Research Paper

\title{
Inhibition of CRL-NEDD8 pathway as a new approach to enhance ATRA-induced differentiation of acute promyelocytic leukemia cells
}

\author{
Shuyuan Liu\#, Jinhua Wan\#, Yunyuan Kong, Yonglu Zhang, Lagen Wan ${ }^{\bowtie}$, Zhanglin Zhang ${ }^{\bowtie}$ \\ Department of Clinical laboratory, The First Affiliated Hospital of Nanchang University, Nanchang, Jiangxi 330006, China \\ \#These authors contributed equally to this work. \\ $\triangle$ Corresponding authors: Zhanglin Zhang, Email: zhzl1984@alumni.sjtu.edu.cn and Lagen Wan, Email: wlgme196412@126.com; Tel: +86-0791-88697032, Mail \\ Address: No. 17 Yongwai Street, Donghu District, Nanchang, Jiangxi, 330006, China. \\ (C) Ivyspring International Publisher. This is an open access article distributed under the terms of the Creative Commons Attribution (CC BY-NC) license \\ (https://creativecommons.org/licenses/by-nc/4.0/). See http://ivyspring.com/terms for full terms and conditions.
}

Received: 2017.11.10; Accepted: 2018.03.02; Published: 2018.04.03

\begin{abstract}
The cullin-RING ligase (CRL)-NEDD8 pathway maintains essential cellular processes, including cell cycle progression, apoptosis, autophagy, DNA repair, antigen processing and signal transduction. Growing evidence demonstrates that the alteration of the CRL-NEDD8 pathway in some cancers constitutes an attractive target for therapeutic intervention, but the roles of CRL-NEDD8 pathway in acute promyelocytic leukemia (APL) is still unclear. In the present study, we found that ATRA could decrease the expression of NEDD8-activating enzyme EI (NAEI) and inhibit the neddylation of cullin1 and cullin3 in the APL cell line NB4. Inactivation of cullin neddylation promoted self-degradation of F-box proteins (Skp2, KLHL20, $\beta \operatorname{TrCP}$ ) and up-regulated the protein expression of $\mathrm{p}^{\mathrm{kip}}$, DEPTOR and DAPK1. MLN4924, a novel inhibitor of NAE1, significantly suppressed cell growth and enhanced apoptosis of APL cells by blocking cullin neddylation and subsequent accumulation of CRL E3 substrates. Furthermore, MLN4924 effectively enhanced ATRA-induced differentiation of APL cells by promoting autophagy. Our findings not only provide further insights into the mechanism of the CRL-NEDD8 axis, but also provide a better understanding of this pathway as a potential target for therapeutic intervention in APL.
\end{abstract}

Key words: ATRA; differentiation; CRL-NEDD8; MLN4924; neddylation

\section{Introduction}

The ubiquitin-proteasome system (UPS) plays a critical role in the degradation of most intracellular proteins. As the largest enzyme family of UPS, the cullin-RING ligases (CRLs) are responsible for ubiquitylation of about $20 \%$ of cellular proteins for targeted degradation[1]. Increasing reports suggest that CRLs are implicated in the regulation of numerous cellular processes such as cell cycle and apoptosis, and aberrant CRL activity is associated with cancers. CRLs are modular assemblies built around a central cullin scaffold, a substrate receptor module and a RING protein that recruits the E2-conjugating enzyme[2]. Pro-degradative activity of CRLs requires modification of cullin by a small ubiquitin-like protein NEDD8[3]. CRL neddylation involves an ordered transfer of NEDD8 by specific NEDD8-activating enzyme E1 (NAE1), NEDD8conjugating enzyme E2 (UBE2M or UBE2F) and NEDD8-E3 ligases[4, 5]. The reverse reaction, deneddylation, catalyzed by the COP9 signalosome (CSN), allows subsequent binding of factors to mediate the disassembly and remodeling of CRL complexes[6, 7]. The binding of NEDD8 to cullin family proteins is required for CRL assembly and activation; however, continuous neddylation of cullins leads to the auto-ubiquitination of CRL subunits followed by degradation[8, 9]. Therefore, CRL-NEDD8 controls a high proportion of 
ubiquitylation events in cells, making this pathway an attractive target for pharmacological manipulation. Recent studies show that retinoic acid-induced gene $G$ (Rig-g), first identified from an APL cell line NB4 treated with ATRA, is able to negatively regulate SCF-E3 ligase activities and largely decrease protein levels of cullin 1 and $\beta$-TrCP, indicating a significant role for inhibition of CRL-NEDD8 pathway in the ATRA-induced APL differentiation[10, 11].

MLN4924, a specific small molecule inhibitor, specifically blocks the activity of NEDD8 E1activating enzyme, efficiently inhibits neddylation of all cullins, resulting in inactivation of CRLs and accumulation of their substrates[12, 13]. It has been shown that MLN4924 has anti-tumor activities both in vitro and in vivo. Treatment of tumor cells (lung cancer, pancreatic cancer, AML, B-cell lymphoma, myeloma) with MLN4924 induces cell cycle arrest, apoptosis and senescence[14-19]. These findings suggested the CRL-NEDD8 pathway as a promising therapeutic target and MLN4924 as a potential drug for cancer therapy.

In this study, we found that ATRA inactivated of CRL1 and CRL3-E3 by inhibiting the neddylation of cullin1 and cullin3 in NB4 cells, then up-regulated the substrate proteins p27kip, DEPTOR and DAPK1. Inhibition neddylation of cullins by MLN4924 significantly suppressed cell growth by inducing $\mathrm{S}$ phase arrest and promoting apoptosis of NB4 cells. Furthermore, we found that MLN4924 effectively enhanced ATRA-induced differentiation of APL cells via promoting autophagy. These data illustrate the important role of CRL-NEDD8 mediated proteolysis in ATRA-induced differentiation of APL, and provide the basis for MLN4924 combined ATRA in the APL therapeutics.

\section{Materials and methods}

\section{Cell culture and reagents}

The APL cell line NB4 was cultured in RPMI 1640 (Gibco BRL, Gaithersburg, MD) containing 10\% FBS, $2 \mathrm{mmol} / \mathrm{L}$ L-glutamine, $10 \mathrm{U} / \mathrm{ml}$ penicillin, and $10 \mu \mathrm{g} / \mathrm{ml}$ streptomycin at $37^{\circ} \mathrm{C}$ in a humidified atmosphere containing 5\% $\mathrm{CO}_{2}$. ATRA (Sigma, St. Louis, MO) and MLN4924 ( MedChemExpress USA ) were dissolved in DMSO to $100 \mathrm{mmol} / \mathrm{L}$ (stock solutions). Protease inhibitors used were PMSF (AMRESCO, Solon, OH) and a cocktail (Roche, Switzerland); they were respectively dissolved in isopropanol and PBS to $100 \mathrm{mmol} / \mathrm{L}$ and 50×. All stock solutions were stored at $-20^{\circ} \mathrm{C}$. Annexin-VFITC/PI kit was purchased from Bestbio Biotechnology (Bestbio, China). Cell cycle detection kit (COULTER DNA PREP reagent kit) was from
Beckman coulter, Inc.

The following primary antibodies were used in this study: rabbit polyclonal anti- Rig-G antibody was described previously[11]; anti-Cul 1 was obtained from Invitrogen (Grand Island, NY); anti-Cul 3 was purchased from BD (Franklin Lakes, NJ); anti-DAPK1 was produced by Sigma (St. Louis, MO); antibodies against LC3, NAE1, p27kip, p-Beclin1 and $\beta \operatorname{TrCP}$ were from Cell Signaling Technologies Inc. (Beverly, MA); anti-NEDD8 was purchased from Abcam (Cambridge, UK); anti-UBE2M and $\beta$-actin were from ABclonal (USA); anti-DEPTOR and Skp2 antibodies, anti-mouse IgG, and anti-rabbit IgG were obtained from Santa Cruz Biotechnology Inc. (USA); anti-Beclin1 and KLHL20 were produced by Abgent (USA).

\section{Cell proliferation and morphology assessment}

The leukemic cells were treated with ATRA or MLN4924 for 1 to 3 days, harvested, and washed in PBS. Then, viable cells were quantified using Cell Counter (Z2, Beckman Coulter), and $4 \times 10^{4}$ viable cells were prepared for cytospin onto glass slides ( $5 \mathrm{~min}$ centrifugation at $500 \mathrm{rpm}$ ). The cells on glass slides were stained with Giemsa (WG16; Sigma-Aldrich, St. Louis, MO) for 5 minutes, rinsed briefly with distilled water, dried, and observed by microscopy.

\section{Detection of the CDI Ib antigen}

Mouse anti-human CD11b-PC5 antibody $(10 \mu \mathrm{l})$ was added to a $100 \mu \mathrm{l}$ cell suspension $\left(\sim 5 \times 10^{5}\right.$ cells $)$ and mixed. The samples were stained for $30 \mathrm{~min}$ at $25^{\circ} \mathrm{C}$, protected from light. After two washes with PBS, cells were fixed with $500 \quad \mu l \quad 2 \%$ paraformaldehyde solution. The expression of the $\mathrm{CD} 11 \mathrm{~b}$ antigen was detected by flow cytometry (FC500, Beckman Coulter).

\section{Analysis of cell cycle and apoptosis}

Cells were treated with or without the drug and cultivated under $37^{\circ} \mathrm{C}$ saturated humidity and $5 \%$ $\mathrm{CO}_{2} .10^{6}$ cells were harvest in the appropriate manner (centrifuged at 2,000 rpm for $5 \mathrm{~min}$ ) and removed the supernatant. For cell cycle analysis, added $50 \mu$ DNA PREP LPR reagent for $1 \mathrm{~min}$ according to the instructions, and then added $300 \mu$ DNA PREPStain reagent and placed it at room temperature for $30 \mathrm{~min}$. Then detected by flow cytometry (FC500, Beckman Coulter) and analyzed cell cycle by MODFIT2 software. For apoptosis analysis, $5 \mu \mathrm{l}$ Annexin-V were added after adding $300 \mathrm{ml}$ Annexin- $\mathrm{V}$ binding solution and placed the mixture at $4^{\circ} \mathrm{C}$ for $15 \mathrm{~min}$. Added $10 \mu \mathrm{PI}$ at indicated time, then analyzed the results by cytometer. Annexin V+ and/or PI+ cells are apoptosis cells. 


\section{Western blot analysis}

Whole cell lysates were prepared with a lysis buffer containing 1\% Triton X-100, $50 \mathrm{mM}$ Tris $(\mathrm{pH}$ 8.0), $150 \mathrm{mM} \mathrm{NaCl}, 1 \mathrm{mM}$ PMSF, $1 \mathrm{mM} \mathrm{Na}_{3} \mathrm{VO}_{4}$, and protease inhibitor cocktail. Protein concentrations were determined using Bio-Rad protein assays. Cell lysate proteins $(50 \mu \mathrm{g})$ were separated on $12 \%$ SDS-PAGE, and electro-transferred to nitrocellulose membranes, which were blocked for 30 minutes at room temperature in Tris-buffered saline- $0.05 \%$ Tween-20 (TTBS) containing 5\% non-fat dry milk. After incubation with TTBS containing primary antibodies for $4 \mathrm{~h}$ at room temperature, membranes were washed $(3 \times 10 \mathrm{~min})$ in TTBS and incubated with peroxidase conjugated secondary antibodies for $1 \mathrm{~h}$. Finally, protein bands were visualized using the enhanced chemiluminescence detection system (Amersham, Piscataway, NJ).

\section{Statistical analysis}

Statistical analysis was performed using Student's t-test. P-values $<0.05$ were considered statistically significant.

\section{Results}

ATRA inactivates cullin 1- and cullin3-mediated CRL-E3 ligases and results in substrate protein accumulation by inhibiting neddylation in NB4 cells

To investigate the effect of ATRA on the activation of CRL-NEDD8 in cultured APL cells, we treated NB4 cells with ATRA $(1 \mu \mathrm{M})$ for 24,48 and 72 hours, and determined the expression levels of cullin1 and cullin3 in NB4 cells. As shown in Fig.1A, western blot analysis of NB4 cells with antibodies against cullin 1 and cullin 3 revealed the dramatic decrease of the neddylated cullin1 and cullin3 band intensity after ATRA treatment for $24 \mathrm{~h}$, but no obvious changes for the un-neddylated cullins. Meanwhile, we determined the expression of Rig-g. The results showed that expression of Rig-g protein started at $72 \mathrm{~h}$, which is significantly delayed than the decrease of cullins. In addition, we assessed the protein levels of two cullin1 F-box proteins, Skp2 and $\beta \operatorname{TrCP}$, and the cullin3 adaptor protein KLHL20 in NB4 cells treated with ATRA. The levels of these three F-box proteins were decreased in a time-dependent manner (Fig. 1B). These results showed that ATRA could inactivate cullin1- and cullin3-mediated E3 ligases, which then inhibited the degradation of substrates such as p27kip, DEPTOR and DAPK1 (Fig. 1C).

We further hypothesized that inactivation of CRLs may be regulated by neddylation in ATRA treated-NB4 cells. We treated NB4 cells with ATRA and monitored NEDD8-modified cullins, NAE1 and UBE2M by western blot assay. The result showed that the proteins of NEDD8-modified cullins, NAE1 and UBE2M were decreased in ATRA-treated NB4 cells (Fig. 1D). Taken together, our data demonstrated that ATRA potently prevented the neddylation of CRLs and trapped them in an inactive state, the respective CRL substrates could not be ubiquitinated and were protected from degradation by the proteasome. These observations suggest that the important role of CRL-NEDD8 mediated proteolysis in ATRA-induced differentiation of APL cells.

\section{Inhibition of neddylation by MLN4924 induces $S$ phage arrest and promotes apoptosis of NB4 cells}

Neddylation contributes to the oncogenic growth of various hematologic malignancies, including acute myeloid leukemia[20]. To investigate effect of CRL-NEDD8 pathway in ATRA-induced differentiation of APL, NB4 cells were treated with varying concentrations of MLN4924 (0, 20, 40, 80 and $160 \mathrm{nM}$ ) for $0,24,48$ and 72 hours. We first determined effect of MLN4924 on cullin neddylation, and the result showed that the neddylated cullin1 was not detectable when treated with 20 nM MLN4924 for 24h (Fig. 2A). Next, we examined the cell proliferation of NB4 cells when treated with MLN4924. As shown in Fig. 2B, MLN4924 inhibited NB4 cells growth in dose- and time-dependent manners. Cell cycle analysis revealed that MLN4924 treatment for 24 hours caused growth arrest at the $S$ phase in a dose-dependent manner (Fig. 2C). Moreover, annexin-V/PI staining assay showed that MLN4924 induced apoptosis of NB4 cells in a dose-dependent manner (Fig. 2D). Collectively, these data demonstrated that MLN4924 potently inhibited cell viability and clonal survival, resulting from induction of $S$ cell cycle arrest and apoptosis.

\section{Inhibition of neddylation by MLN4924 enhances ATRA-induced differentiation of NB4 cells}

In order to explore the effects of CRL-NEDD8 pathway on ATRA-induced differentiation, we suppressed neddylation by MLN4924 and assessed the myeloid differentiation by measuring the expression of granulo-monocytic differentiation marker CD11b. As the result seen in Fig. 3A, $40 \mathrm{nM}$ MLN4924 induced the CD11b expression in a timedependent manner. Next, we tested the differentiation of NB4 cells when treated with ATRA combined with MLN4924 (40nM) for 48 hours, we found that the expression of $\mathrm{CD} 11 \mathrm{~b}$ increased to $84.5 \%$ when treated with $1 \mu \mathrm{M}$ ATRA, and $68.5 \%$ with $0.01 \mu \mathrm{M}$ ATRA. The 
percentage of CD11b-positive cells reached to $96.3 \%$ when treated with $1 \mu \mathrm{M}$ ATRA combined with MLN4924, and $88.1 \%$ for the treatment of $0.01 \mu \mathrm{M}$ ATRA combined with MLN4924 (Fig. 3B). Cell morphology data also demonstrated inhibition of neddylation by MLN4924 could enhance ATRA-induced differentiation of NB4 cells (Fig. 3C).

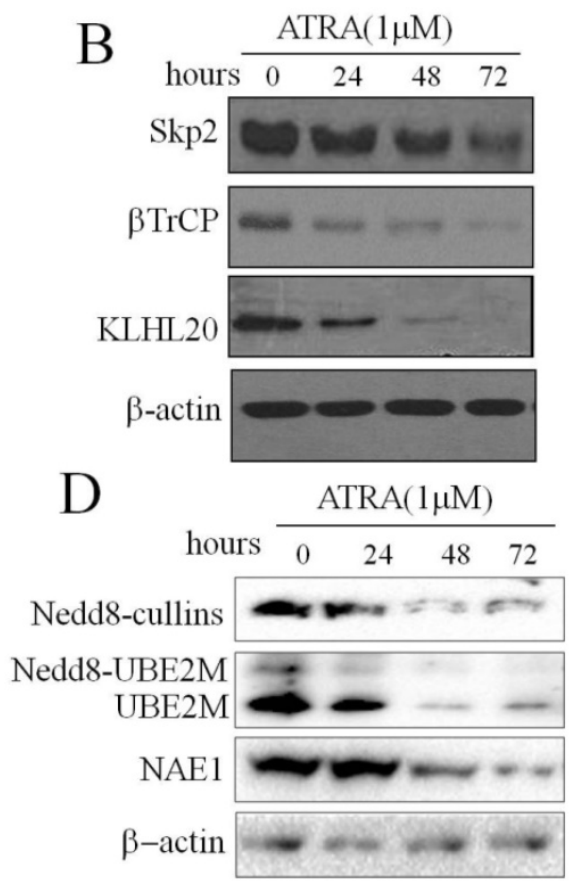

Figure 1. ATRA inhibits cullin 1- and cullin3-mediated CRL-E3 ligases and results in substrate protein accumulation in NB4 cells. Effect on CRL components and substrates after treatment of NB4 cells with $1 \mu$ M ATRA for 0, 24, 48 and 72 hours. (A) Immunoblotting for cullin 1, cullin 3 and Rig-G. (B) For two cullin1 F-box proteins, Skp2 and $\beta \operatorname{TrCP}$, and the cullin3 adaptor protein KLHL20. (C) For the SCFskp2 substrate p27kip, SCF $\beta$ TrCP substrate DEPTOR and cullin3-CRL substrate DAPK1. (D) For E1-activating enzyme NAE1 and E2-conjugating enzyme UBE2M. The expression of $\beta$-actin was used as loading control.

A

C hours $\frac{\text { ATRA }(1 \mu \mathrm{M})}{0 \quad 24 \quad 48 \quad 72}$

DEPTOR $=\ldots$

p27kip $\cdots-\cdots$

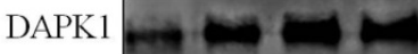

$\beta$-actin $-\infty$

MLN4924

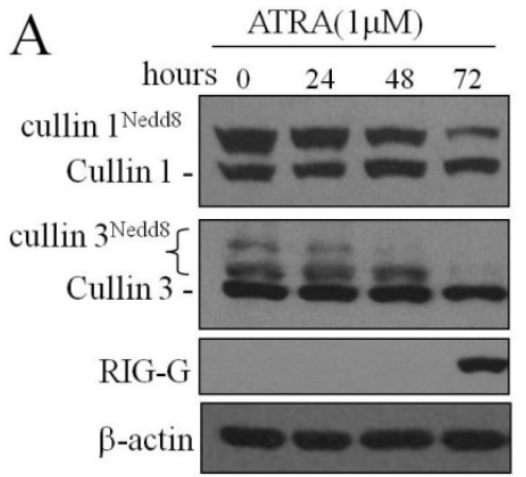

B

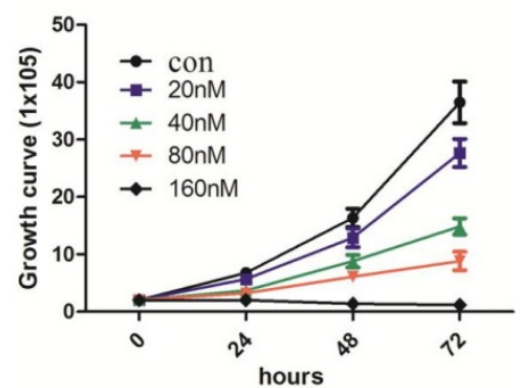

$\mathrm{C}$

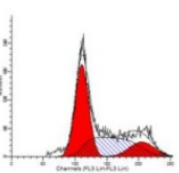

con

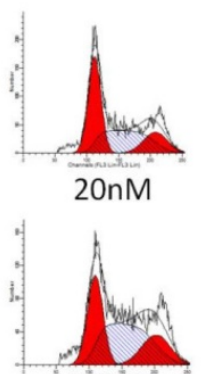

$80 \mathrm{nM}$

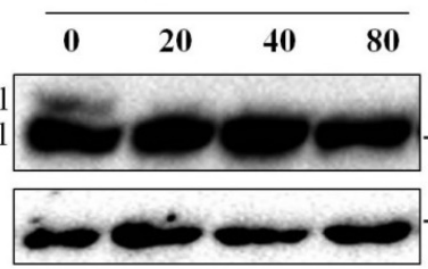

$-86 \mathrm{kD}$

$42 \mathrm{kD}$

$\mathrm{D}$
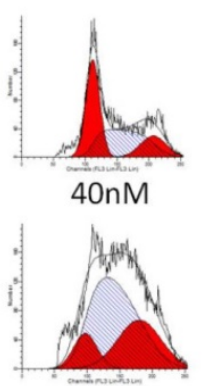

$160 \mathrm{nM}$

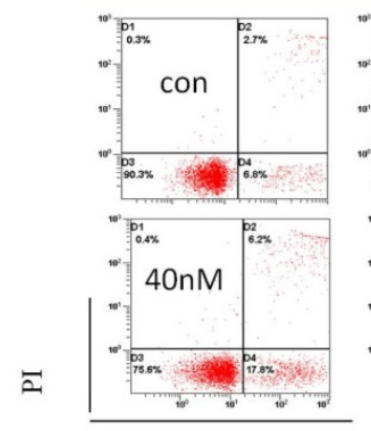

Annexin V

Figure 2. Inhibition of neddylation by MLN4924 induce S phage arrest and promotes apoptosis of NB4 cells. (A) NB4 cells were treated with MLN4924 (0, 20, 40 and $80 \mathrm{nM})$ for $0,24,48$ and 72 hours, and the protein levels of cullin 1 were detected by western blotting, with $\beta$-actin used as loading control. (B) NB4 cells were exposed to MLN4924 (0,20,40,80 and $160 \mathrm{nM})$, the growth curve was formed. (C) NB4 cells were treated with MLN4924 for 48 h, stained with $\mathrm{PI}$, and examined with flow cytometry assays. (D) NB4 cells were treated with MLN4924 for 48 h, stained with Annexin-V-FITC and PI, and examined with flow cytometry assays. 

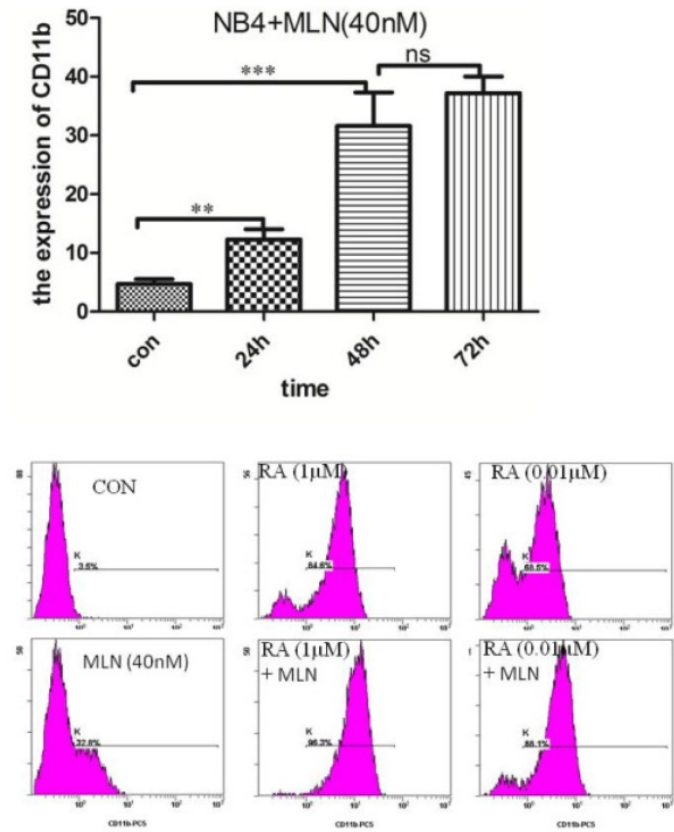

MLN4924(40nM)
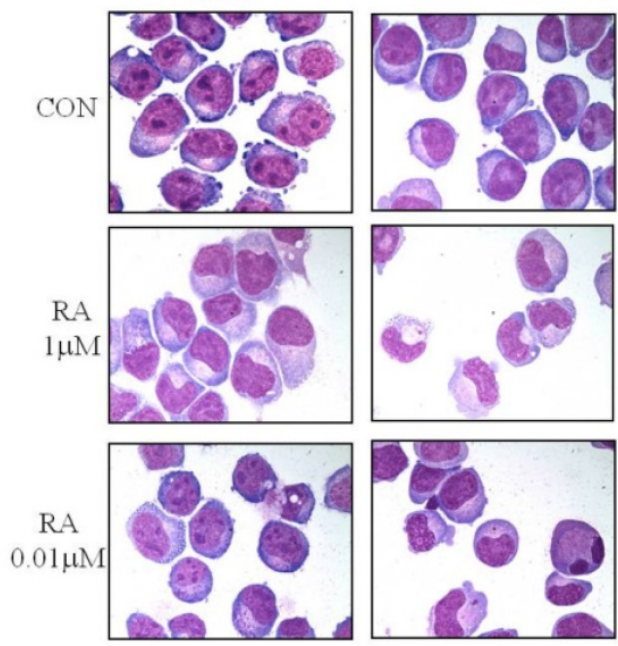

Figure 3. MLN4924 promotes the expression of CDI l b and enhances ATRA induced differentiation of NB4 cells. (A) The myeloid differentiation antigen CD1 lb was measured by flow cytometry in NB4 cells after treatment with MLN4924 (40 nM) for 0, 24, 48 and 72 hours. (B) NB4 cells were treated with ATRA $(0.01 \mu \mathrm{M})$ and/or MLN4924 $(40 \mathrm{nM})$ for $48 \mathrm{~h}$, and the expression of CD1 lb was measured by FCM. (C) Giemsa staining of NB4 cells treated with ATRA (0.01 $\mu \mathrm{M})$ and/or MLN4924 (40 nM). Graphical data indicates the mean \pm S.E.M. ns represent having no statistics, $* *$ and $* * *$ indicate less than 0.01 and 0.005 of $\mathrm{P}$-values.

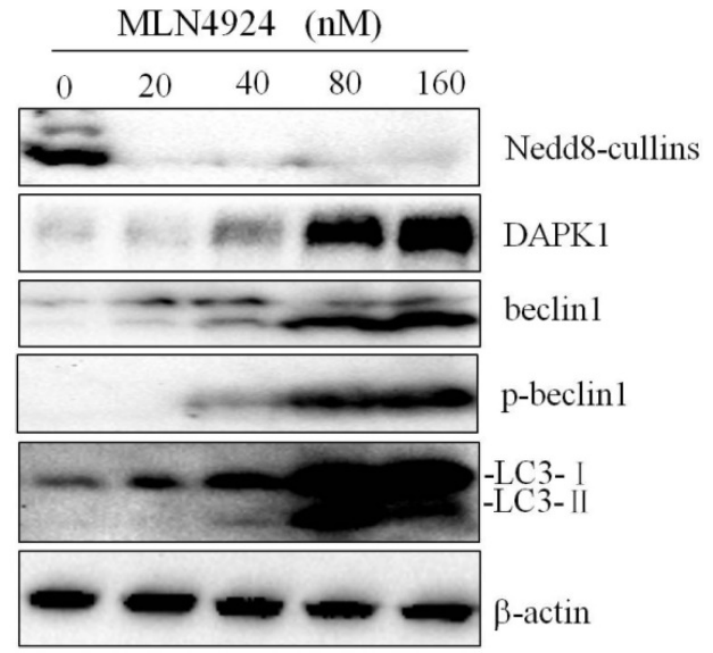

Figure 4. Inhibition of neddylation by MLN4924 induced autophagy by up-regulating DAPK1 and Beclin I. NB4 cells were treated with MLN4924 (0, 20, 40, 80 and $160 \mathrm{nM})$ for $24 \mathrm{~h}$, and the levels of Nedd8, DAPK1, Beclin 1, p-Beclin 1 and LC3 were examined by Western blot. $\beta$-actin was used as loading control.

\section{Inhibition of neddylation by MLN4924 induced autophagy by up-regulating DAPK 1 and Beclin 1}

As an important cellular response, autophagy plays a key role in the regulation of cell survival during cellular stresses. Some studies show that MLN4924 effectively induces autophagy in multiple human cancer lines, indicating a general phenom- enon. Furthermore, we explored whether MLN4924 affects NB4 cell autophagy. Autophagy in NB4 cells treated with MLN4924 (0, 20, 40, 80 and $160 \mathrm{nM})$ for 24 hours was detected via testing the conversion of LC3-I to LC3-II by western blot. As shown in Fig. 4, the conversion of LC3-I to LC3-II was increased by treatment with MLN4924 and this increase was dose-dependent. Western blot results suggested that MLN4924 could inhibit the neddylation of cullins, and up-regulated DAPK1, Beclin1 and p-Beclin1. Taken together, the data show that MLN4924 could induced autophagy by up-regulating DAPK1 and Beclin1.

\section{Inhibition of neddylation by MLN4924 enhances ATRA-induced autophagy}

Recent studies have shown that autophagy promotes degradation of the PML/RARa fusion protein and contributes to ATRA induced differentiation of NB4 cells, and CRLs could control autophagy through modification of regulators of autophagy such as DAPK1[21, 22]. We treated NB4 cells with $0.01 \mu \mathrm{M}$ ATRA in combination with 40nM MLN4924, and analyzed the expression of autophagy related proteins by western blot. As shown in Fig. 5A, MLN4924 treatment alone inhibited cullin1 and cullin3 neddylation, demonstrating the inactivation of neddylation pathway. In comparison with MLN4924 alone, MLN4924+ATRA treatment induced significant up-regulation of autophagy related proteins including DAPK1 and p-Beclin1 (Fig. 5B). 
Interesting, the level of Belin1 in these two group were similar. We also found that MLN4924 combined with ATRA treatment in NB4 cells caused more obvious up-regulation of LC3-II level, indicating that MLN4924 could enhance ATRA-induced autophagy in APL cell line NB4.

\section{Discussion}

Although great achievements have been made in understanding the mechanistic basis for ATRAinduced differentiation, some other genes may also contribute to the treatment of APL. It is generally considered that Rig-g inhibits the proliferation and propels the ATRA-induced differentiation of NB4 cells, and its expression level is related to the morbid state of APL patients[23, 24]. In the present study, we found that the expression of neddylated cullin1 and cullin3 dramatically decreased at $24 \mathrm{~h}$ in ATRAtreated NB4 cells, while Rig-G expression decreased at $72 \mathrm{~h}$, which is significantly delayed than the decrease of cullins. These results indicated that other mechanisms also contribute to the ATRA-induced differentiation, and cullin-RING ligase may play an important role in the course.

Recent studies have clearly shown that cullin-RING ligase and neddylation pathway are over-activated in various human cancers[17, 25]. CRLs are multi-protein complexes assembled in mammals on seven cullin scaffoles (cullin 1, 2, 3, 4a, $4 \mathrm{~b}, 5$ and 7). In the cell, the activities of CRLs can be regulated by the ratio of NEDD8 linkage to the cullin proteins. The binding of NEDD8 to cullin family proteins (neddylation) is required for CRL assembly and activation. Upregulation of CRL-NEDD8 may contribute to tumorigenesis, unrestrained cell proliferation and resistance to apoptosis in cancer.
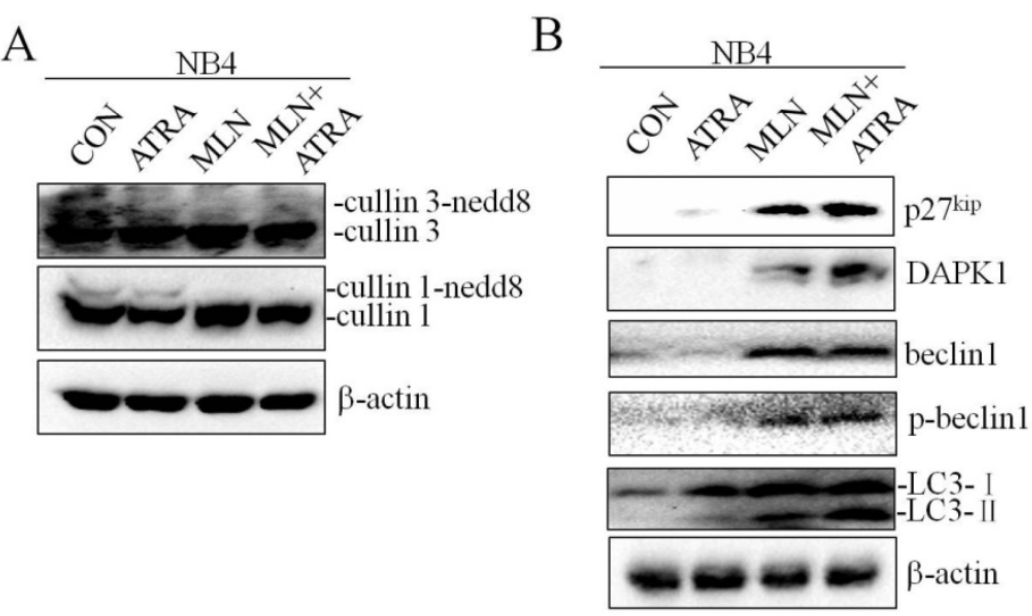

Figure 5. Inhibition of neddylation by MLN4924 enhance ATRA induced autophagy. NB4 cells were treated with ATRA $(0.01 \mu \mathrm{M})$ and/or MLN4924 $(40 \mathrm{nM})$ for $24 \mathrm{~h}$, and the expression of cullin3, cullin 1, p27kip, DAPK1, Beclin 1, p-Beclin 1 and LC3 were analyzed by Western blot. The expression of $\beta$-actin was used as loading control.
CRL-NEDD8 pathway has emerged as one of the potential cancer targets[17, 25, 26]. In the present study, we found that ATRA inactivated CRL1 and CRL3-E3 by inhibiting the neddylation of cullin1 and cullin3 in NB4 cells, which then up-regulated the substrate proteins p27kip, DEPTOR and DAPK1. To evaluate whether and how CRL-NEDD8 pathway is involved in ATRA-induced differentiation, MLN4924, a novel inhibitor of NAE1, has been used as single agent or in combination with ATRA on APL cell line NB4. The results showed that MLN4924 treatment inhibited the cell proliferation by inducing cell cycle arrest at the S phase in NB4 cells, potently suppressed cell viability and clonal survival. More importantly, the combining MLN4924 with ATRA enhanced cell differentiation. It should be noted that we used a lower dose of ATRA $(0.01 \mu \mathrm{M})$ than previously reported, and this low dose of ATRA combining with MLN4924 could significantly induce cell differentiation efficiently. To elucidate the underlying mechanism, we focused on potential changes involved in the processes of ATRA treatment.

It has been reported that ATRA stimulates the mTOR-dependent autophagy, which contributes to therapy-induced degradation of the PML-RARa[21]. Inhibiting autophagy blocked PML-RARa degradation and subsequently granulocytic differentiation of human myeloid leukemic cells, demonstrating a role for autophagy in ATRA-induced APL differentiation $[21,27]$. In addition, it has been shown that the cul3-KLHL20 E3 ligase regulated autophagy by impacting DAPK1 protein degradation. DAPK phosphorylates Beclin-1 on Thr119 located at a crucial position in its $\mathrm{BH} 3$ domain, and thus promoted the dissociation of Beclin-1 from its inhibitor Bcl- $\mathrm{X}_{\mathrm{L}}$, resulting in the induction of autophagy[28, 29]. Our results presented above showed MLN4924 combined with ATRA treatment caused more significantly accumulation of DAPK1 and p-Beclin1 and up-regulation of LC3-II level, suggesting that inhibition of neddylation by MLN4924 may enhance ATRA-induced differentiation of NB4 cells, through triggering cell autophagy via accumulated DAPK1 and Beclin1. Meanwhile, Zhao et al showed that MLN4924 induced protective autophagy through inducing accumulation of SCF E3 substrates DEPTOR, a direct inhibitor of mTORC1 and the HIF1-REDD1-TSC1 axis, a negative regulatory pathway of mTORC1[30]. We noted that ATRA could inhibite the degradation of substrates and induce the accumulation of the mTOR-inhibitory 
protein DEPTOR, revealing that MLN4924-enhanced differentiation may be attributed to blockage of mTOR signals via DEPTOR.

In summary, we demonstrate here that inhibition of neddylation by MLN4924 significantly suppress APL cell growth by blocking cullin neddylation and subsequent accumulation of CRL E3 substrates, which trigger cell cycle regulation and apoptosis, and MLN4924 can induce autophagy by DAPK1 accumulation and effectively enhance ATRA-induced differentiation of APL cells (Fig 6). Our findings not only provide further insights into the mechanism of the CRL-NEDD8 axis, but also contribute to a better understanding of this pathway as a potential target for therapeutic intervention in APL. Furthermore, it is of interest to develop complementary treatment strategies for APL including CRL-NEDD8 inhibitors which increase the sensitivity of APL cell to ATRA action.

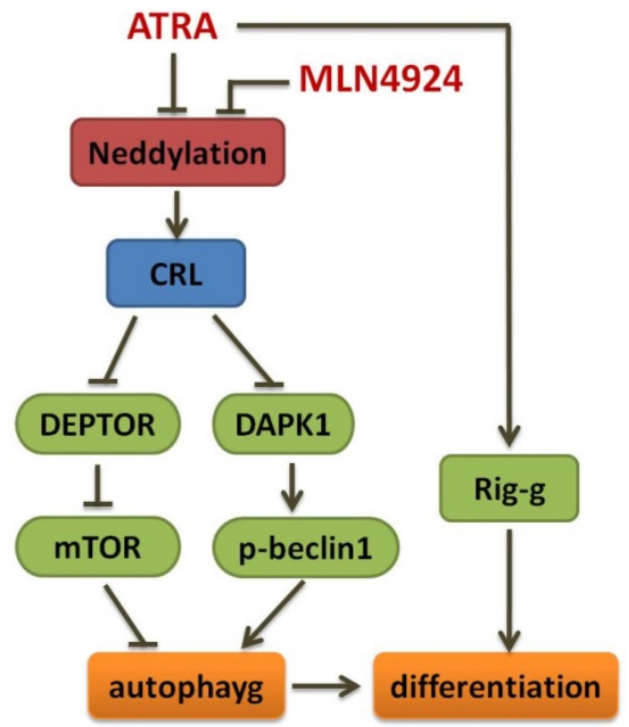

Figure 6. Schema of the mechanism for MLN4924 enhancing ATRA-induced differentiation.

\section{Acknowledgements}

We thank all members of Department of clinical laboratory of the First Affiliated Hospital of Nanchang University for their support. This work was supported by Natural Science Foundation of China (81760539), Natural Science Foundation of Jiangxi Province (20151BAB205020) and Science and Technology Plan Project of Jiangxi Provincial Health Planning Commission (20171045).

\section{Author Contributions}

Zhanglin Zhang contributed to the study design. Shuyuan Liu, Jinhua Wan, Yunyuan Kong, Yonglu Zhang, Lagen Wan, Zhanglin Zhang preformed the research and conducted the data analysis. Zhanglin Zhang and Lagen Wan wrote the manuscript.

\section{Competing Interests}

The authors have declared that no competing interest exists.

\section{References}

1. Deshaies RJ, Joazeiro CA: RING domain E3 ubiquitin ligases. Annu Rev Biochem 2009, 78:399-434.

2. Zheng N, Schulman BA, Song L, Miller JJ, Jeffrey PD, Wang P, Chu C, Koepp DM, Elledge SJ, Pagano $M$ et al: Structure of the Cul1-Rbx1-Skp1-F boxSkp2 SCF ubiquitin ligase complex. Nature 2002, 416(6882):703-709.

3. Enchev RI, Schulman BA, Peter M: Protein neddylation: beyond cullin-RING ligases. Nat Rev Mol Cell Biol 2015, 16(1):30-44.

4. Deshaies RJ, Emberley ED, Saha A: Control of cullin-ring ubiquitin ligase activity by nedd8. Subcell Biochem 2010, 54:41-56.

5. Duda DM, Scott DC, Calabrese MF, Zimmerman ES, Zheng N, Schulman BA: Structural regulation of cullin-RING ubiquitin ligase complexes. Curr Opin Struct Biol 2011, 21(2):257-264.

6. Pierce NW, Lee JE, Liu X, Sweredoski MJ, Graham RL, Larimore EA, Rome M, Zheng N, Clurman BE, Hess S et al: Cand1 promotes assembly of new SCF complexes through dynamic exchange of F box proteins. Cell 2013, 153(1):206-215.

7. Cope GA, Deshaies RJ: COP9 signalosome: a multifunctional regulator of SCF and other cullin-based ubiquitin ligases. Cell 2003, 114(6):663-671.

8. Wee S, Geyer RK, Toda T, Wolf DA: CSN facilitates Cullin-RING ubiquitin ligase function by counteracting autocatalytic adapter instability. Nat Cell Biol 2005, 7(4):387-391

9. Wu JT, Chan YR, Chien CT: Protection of cullin-RING E3 ligases by CSN-UBP12. Trends Cell Biol 2006, 16(7):362-369.

10. Xiao S, Li D, Zhu HQ, Song MG, Pan XR, Jia PM, Peng LL, Dou AX, Chen GQ, Chen SJ et al: RIG-G as a key mediator of the antiproliferative activity of interferon-related pathways through enhancing p21 and p27 proteins. Proc Natl Acad Sci U S A 2006, 103(44):16448-16453.

11. Xu GP, Zhang ZL, Xiao S, Zhuang LK, Xia D, Zou QP, Jia PM, Tong JH: Rig-G negatively regulates SCF-E3 ligase activities by disrupting the assembly of COP9 signalosome complex. Biochem Biophys Res Commun 2013, 432(3):425-430.

12. Soucy TA, Smith PG, Milhollen MA, Berger AJ, Gavin JM, Adhikari S, Brownell JE, Burke KE, Cardin DP, Critchley S et al: An inhibitor of NEDD8-activating enzyme as a new approach to treat cancer. Nature 2009, 458(7239):732-736.

13. Brownell JE, Sintchak MD, Gavin JM, Liao H, Bruzzese FJ, Bump NJ, Soucy TA, Milhollen MA, Yang X, Burkhardt AL et al: Substrate-assisted inhibition of ubiquitin-like protein-activating enzymes: the NEDD8 E1 inhibitor MLN4924 forms a NEDD8-AMP mimetic in situ. Mol Cell 2010, 37(1):102-111.

14. Blank JL, Liu XJ, Cosmopoulos K, Bouck DC, Garcia K, Bernard H, Tayber O, Hather G, Liu R, Narayanan U et al: Novel DNA damage checkpoints mediating cell death induced by the NEDD8-activating enzyme inhibitor MLN4924. Cancer Res 2013, 73(1):225-234.

15. Godbersen JC, Humphries LA, Danilova OV, Kebbekus PE, Brown JR, Eastman A, Danilov AV: The Nedd8-activating enzyme inhibitor MLN4924 thwarts microenvironment-driven NF-kappaB activation and induces apoptosis in chronic lymphocytic leukemia B cells. Clin Cancer Res 2014, 20(6):1576-1589.

16. Jia L, Li H, Sun Y: Induction of p21-dependent senescence by an NAE inhibitor, MLN4924, as a mechanism of growth suppression. Neoplasia 2011, 13(6):561-569.

17. Li L, Wang M, Yu G, Chen P, Li H, Wei D, Zhu J, Xie L, Jia H, Shi J et al: Overactivated neddylation pathway as a therapeutic target in lung cancer. $J$ Natl Cancer Inst 2014, 106(6):dju083.

18. Lin JJ, Milhollen MA, Smith PG, Narayanan U, Dutta A: NEDD8-targeting drug MLN4924 elicits DNA rereplication by stabilizing Cdt1 in S phase, triggering checkpoint activation, apoptosis, and senescence in cancer cells. Cancer Res 2010, 70(24):10310-10320.

19. Luo Z, Yu G, Lee HW, Li L, Wang L, Yang D, Pan Y, Ding C, Oian J, Wu L et al: The Nedd8-activating enzyme inhibitor MLN4924 induces autophagy and apoptosis to suppress liver cancer cell growth. Cancer Res 2012, 72(13):3360-3371.

20. Swords RT, Kelly KR, Smith PG, Garnsey JJ, Mahalingam D, Medina E, Oberheu K, Padmanabhan S, O'Dwyer M, Nawrocki ST et al: Inhibition of NEDD8-activating enzyme: a novel approach for the treatment of acute myeloid leukemia. Blood 2010, 115(18):3796-3800.

21. Isakson $\mathrm{P}$, Bjoras $\mathrm{M}$, Boe SO, Simonsen A: Autophagy contributes to therapy-induced degradation of the PML/RARA oncoprotein. Blood 2010, 116(13):2324-2331.

22. Cui D, Xiong X, Zhao Y: Cullin-RING ligases in regulation of autophagy. Cell Div 2016, 11:8 
23. Lou YJ, Pan XR, Jia PM, Li D, Xiao S, Zhang ZL, Chen SJ, Chen Z, Tong JH: IRF-9/STAT2 [corrected] functional interaction drives retinoic acid-induced gene G expression independently of STAT1. Cancer Res 2009, 69(8):3673-3680.

24. Lou YJ, Pan XR, Jia PM, Jin J, Tong JH: RIG-G inhibits the proliferation of NB4 cells and propels ATRA-induced differentiation of APL cells. Leuk Res 2016, 40:83-89.

25. Soucy TA, Dick LR, Smith PG, Milhollen MA, Brownell JE: The NEDD8 Conjugation Pathway and Its Relevance in Cancer Biology and Therapy. Genes Cancer 2010, 1(7):708-716.

26. Milhollen MA, Narayanan U, Soucy TA, Veiby PO, Smith PG, Amidon B: Inhibition of NEDD8-activating enzyme induces rereplication and apoptosis in human tumor cells consistent with deregulating CDT1 turnover. Cancer Res 2011, 71(8):3042-3051.

27. Orfali N, O'Donovan TR, Nyhan MJ, Britschgi A, Tschan MP, Cahill MR, Mongan NP, Gudas LJ, McKenna SL: Induction of autophagy is a key component of all-trans-retinoic acid-induced differentiation in leukemia cells and a potential target for pharmacologic modulation. Exp Hematol 2015, 43(9):781-793 e782.

28. Zalckvar E, Berissi H, Eisenstein M, Kimchi A: Phosphorylation of Beclin 1 by DAP-kinase promotes autophagy by weakening its interactions with $\mathrm{Bcl}-2$ and Bcl-XL. Autophagy 2009, 5(5):720-722.

29. Zalckvar E, Berissi H, Mizrachy L, Idelchuk Y, Koren I, Eisenstein M, Sabanay H, Pinkas-Kramarski R, Kimchi A: DAP-kinase-mediated phosphorylation on the $\mathrm{BH} 3$ domain of beclin 1 promotes dissociation of beclin 1 from $\mathrm{Bcl}-\mathrm{XL}$ and induction of autophagy. EMBO Rep 2009, 10(3):285-292.

30. Zhao Y, Xiong X, Jia L, Sun Y: Targeting Cullin-RING ligases by MLN4924 induces autophagy via modulating the HIF1-REDD1-TSC1-mTORC1DEPTOR axis. Cell Death Dis 2012, 3:e386. 